\title{
Elicitation of Expert Knowledge to Inform Object-Based Audio Rendering to Different Systems
}

\author{
JAMES WOODCOCK', WILLIAM J. DAVIES ${ }^{1}$, FRANK MELCHIOR ${ }^{2}$, AND TREVOR J. COX, ${ }^{1}$ AES Member
}

\author{
${ }^{1}$ Acoustics Research Centre, University of Salford, Salford, M5 4WT, United Kingdom \\ ${ }^{2}$ BBC R\&D, Dock House, MediaCityUK, Salford, M50 2LH, United Kingdom
}

\begin{abstract}
Object-based audio presents the opportunity to optimize audio reproduction for different listening scenarios. Vector base amplitude panning (VBAP) is typically used to render objectbased scenes. Optimizing this process based on knowledge of the perception and practices of experts could result in significant improvements to the end user's listening experience. An experiment was conducted to investigate how content creators perceive changes in the perceptual attributes of the same content rendered to systems with different numbers of channels, and to determine what they would do differently to standard VBAP and matrix based downmixes to minimize these changes. Text mining and clustering of the content creators' responses revealed six general mix processes: the spatial spread of individual objects, EQ and processing, reverberation, position, bass, and level. Logistic regression models show the relationships between the mix processes, perceived changes in perceptual attributes, and the rendering method/speaker layout. The relative frequency of use for the different mix processes was found to differ between categories of audio objects suggesting that any downmix rules should be object category specific. These results give insight into how object-based audio can be used to improve listener experience and provide the first template for doing this across different reproduction systems.
\end{abstract}

\section{INTRODUCTION}

Object-based broadcast has been described as the "logical next step" in broadcast technology [1]; this is reflected in current large scale research projects [2-4], standardization activities [5, 6], interest from broadcasters [1], and commercialization [7, 8]. Object-based audio (OBA) is an approach to sound storage, transmission, and reproduction whereby individual audio objects with associated metadata are transmitted and rendered at the client side of the broadcast chain. An object will typically consist of an audio signal and metadata indicating the object's position and level; objects may also contain semantic metadata indicating, for example, the language of a dialogue track or whether the object is positioned on or off screen. This is in contrast to traditional channel based audio, where pre-rendered content for a fixed reproduction system is broadcast. One of the main benefits of OBA is that the content can be rendered optimally for the given reproduction setup, environment, or listening context. For example, the same audio content could be rendered over loudspeakers, mobile devices, or binaurally over headphones for a VR. However, there are many open questions regarding how to render OBA content optimally for different reproduction systems.

Panning in object-based systems is generally done using vector base amplitude panning (VBAP) [9]. As objectbased rendering algorithms have knowledge of the target reproduction setup, there is an opportunity to further optimize the reproduction via metadata adaptation. For example, the panning trajectories of objects in the scene could be modified depending on the limitations of the target loudspeaker layout. This could lead to significant improvements in listener experience over traditional VBAP rendering, particularly to systems with few channels, such as two-channel stereo, where VBAP configurations are not applicable. Optimization of object-based rendering could be based on objective soundfield parameters of the scene (e.g., aiming for an equal distribution of energy from all directions), perceptual parameters of the scene (e.g., by trying to maximize listener envelopment), or could aim to simulate what an expert would do when mixing object-based content for different speaker layouts (e.g., rules describing level adjustments for individual objects). This paper is concerned with the latter, and aims to develop a framework to build expert 
knowledge into object-based rendering. The presented results provide the first evidence of what expert mixers would do when mixing object-based audio for different systems and improves confidence that OBA can deliver on promises of format agnosticism and improved listener experience.

\subsection{Intelligent Systems in Audio Production}

There have been a number of investigations into intelligent systems for audio production. These systems include automatic microphone mixing $[10,11]$, automatic panning algorithms [12], gain normalization algorithms for feedback prevention [13], algorithms designed to reduce masking [14], automatic equalization [15], and automatic music mixing $[16,17]$. Generally, these systems operate on low level signal and psychoacoustic features.

Ren et al. [18] proposed an automatic music production system based on embedded expert knowledge. The system extracts a feature set based on the musical score such as pitch and marks of expression, musical features such as loudness and pitch deviation, and technical audio features such as signal to noise ratio. These features are then used to model the probability of different processes being applied to the production. Pestana and Reiss [19] used a grounded theory approach to investigate current practices in mix engineering to inform intelligent production strategies. This study included an extensive literature search, along with interviews with a large panel of successful mix engineers. The main outcome of this work was a set of rules related to mixing best practices.

The systems described in this section have generally been designed for music applications and operate within a channel-based framework. To date there has been no investigation into how professionals mix non-musical objectbased content for 3D audio systems. The results presented in this paper, therefore, provide data that will be useful in bringing together object-based audio and intelligent systems in audio production.

\subsection{Perception of Spatial Audio}

There have been numerous studies that have aimed to elicit perceptual attributes for reproduced sound [20-29]. Generally, the outcome of these studies is a non-orthogonal set of attributes that can be used to rate perceptual differences between different reproduction conditions (loudspeaker layouts, rendering methods, etc.). Generally, these attribute sets describe various timbral (such as clarity and coloration) and spatial (such as envelopment and horizontal width) perceptual attributes. An overview of these studies is given by Francombe et al. [30].

Recently, Francombe et al. [31,32] conducted a study to determine which perceptual attributes contribute to the listener preference of reproduced audio. Their study included state of the art reproduction methods including systems with height such as $4+5+0$ and $9+10+3$ speaker layouts ${ }^{1}$. An elicitation experiment was conducted with a group of

\footnotetext{
${ }^{1}$ The convention for describing loudspeaker layouts from [33] is used in this paper. $N_{U}+N_{M}+N_{B}$ describes the number of
}

expert and non-expert listeners that resulted in two sets of attributes (one for each of the listener groups) covering a range of timbral and spatial attributes. This attribute set is utilized in the present study (see Sec. 2.3).

The studies described in this section provide useful tools to assess the perception of reproduced sound. However, little is known about how perceptual attributes are degraded when the same object-based content is rendered to systems with different numbers of channels and how professional mix engineers would alter the downmix to compensate for these changes.

\subsection{Conceptual Model}

Conceptually, the work presented in this paper assumes the following model of perception. It is assumed that when object-based audio is rendered to different loudspeaker layouts, changes in the physical sound field will cause a change in the auditory scene perceived by the listener. According to Coombs [34], differences between pairs of stimuli represent a distance in a latent high dimensional psychological space, which can be described by a finite number of perceptual dimensions. In the perception of complex sound stimuli, these dimensions are assumed to relate to some combination of perceptual attributes [35]. Therefore, if a sound designer is tasked to remix content to a different loudspeaker layout with the aim of preserving the production intent of the original content, their judgments of similarity between the original and the re-rendered content will be based on differences in the positions of the stimuli in this perceptual space. When a change in these perceptual attributes is detected by the sound designer, they will make changes to the mix of the re-rendered content to attempt to compensate for the change in the perceptual attribute. It is this relationship between perceptual attributes and general mix processes that is of interest in this paper.

\subsection{Aims and Objectives}

At present, there has been no systematic investigation of how professionals mix object-based content for different reproduction systems or how perceptual attributes change when object-based content is rendered to different loudspeaker systems. This paper reports on an experiment that was conducted to investigate:

1. Which perceptual attributes change when the same object-based content is rendered to systems with different numbers of channels, and;

2. What professional content creators would do differently to a standard VBAP renderer when content is rendered to systems with different numbers of channels.

The first objective of this exploratory study is to identify a small number of the most common mix processes used by professionals when remixing object-based content

loudspeakers in the upper layer $\left(N_{U}\right)$, middle layer $\left(N_{M}\right)$, and bottom layer $\left(N_{B}\right)$. 
for systems with different numbers of channels. A second objective is to explore how the perceptual attributes of the rendered soundfield change between VBAP renderings of the same content to different speaker layouts. The identified mix processes can be used by designers of OBA systems and by researchers in OBA, intelligent audio systems, and spatial audio generally. The processes will also inform future research, the aim of which will be to determine quantitative rules that can be used when rendering OBA for different loudspeaker layouts.

\subsection{Structure of Study}

The work presented in this paper consists of three main phases. First, experiments were conducted with professional audio content producers in which they were asked to compare a 9+10+5 channel rendering of an object-based audio drama scene to a rendering of the same content to a system with fewer channels. The content producers were asked what they would do differently if they had control over mix for the system with fewer channels and indicate what perceptual attributes changed between the reference and the lower channel version. As this is an exploratory study, aiming to elicit the common mix processes a content producer might employ when remixing object-based content for loudspeaker systems with different numbers of channels, the test participants were asked openly about their intentions rather than asking them to directly operate on the mix. A follow-up experiment will give the content producers direct control over a small number of the most commonly suggested processes to determine quantitative downmix rules for those processes.

The open text data collected in the first phase were analyzed using text mining techniques and follow-up interviews with the test participants to identify a small number of common changes suggested by the sound designers. Finally, the relationships between the data relating to changes in perceptual attributes, the identified mix processes, and the rendering method/speaker layout were explored using logistic regression models.

\section{MATERIALS AND METHODS}

\subsection{Ethics}

The experiment described in this paper was approved by the University of Salford ethics committee. Participants took part in the experiment voluntarily, and written consent was taken prior to the test session. Participants were free to withdraw from the experiment at any time without needing to give a reason to the researcher.

\subsection{Stimuli}

Stimuli for the experiment were generated using professionally produced object-based audio drama content, produced as part of the S3A project [36]. The clips were originally mixed for a $9+10+5$ channel setup $(9+10+3$ as specified in Recommendation ITU-R BS.2051 [33] with 2 additional speakers in the lower layer at $\pm 135^{\circ}$ ); subwoofer signals in the listening room during the mix were generated
Table 1. Description of stimuli used in the listening experiment. The scenes are available from https://doi.org/10.17866/rd.salford.3120112.

\begin{tabular}{|c|c|c|}
\hline Scene & Description & Main features \\
\hline 1 & $\begin{array}{l}\text { Children run around the } \\
\text { listener in a forest }\end{array}$ & $\begin{array}{l}\text { 3D diffuse background and } \\
\text { music; Movement in } \\
\text { lower layer (footsteps); } \\
\text { Elevated narrator }\end{array}$ \\
\hline 2 & Within a crowd at a protest & $\begin{array}{l}\text { Surrounding crowd; } \\
\text { Elevated static source; } \\
\text { Dialogue to the side of } \\
\text { listener }\end{array}$ \\
\hline 3 & $\begin{array}{l}\text { A child runs down stairs } \\
\text { behind the listener and } \\
\text { speaks with his family }\end{array}$ & $\begin{array}{l}\text { Movement from behind; } \\
\text { Multispeaker dialogue }\end{array}$ \\
\hline 4 & $\begin{array}{l}\text { A large monster approaches } \\
\text { from behind and leaps } \\
\text { over the listener's head }\end{array}$ & $\begin{array}{l}\text { Movement over listener's } \\
\text { head; Composite object } \\
\text { made of different objects; } \\
\text { Movement in lower layer }\end{array}$ \\
\hline
\end{tabular}

by an IOSONO Core bass management system. Four clips, each with a duration of around 20 seconds, were selected from this material to be used in the test. The clips were selected to demonstrate a wide range of features of advanced $3 \mathrm{D}$ audio systems and to include a range of different object types [37, 38]. Descriptions of the four clips are provided in Table 1.

Object-based renderings of the clips were generated for $4+9+0,0+5+0$, and $0+2+0$ speaker layouts (as specified in Recommendation ITU-R BS.2051 [33]) using VBAP. These systems were selected to include a 3D surround layout, a 2D surround layout, and a standard stereo layout. The renderings were generated from the original object-based scenes, using the original metadata to define the position of sources. In the case of the 2D systems, the height information was disregarded in the rendering. As VBAP is not able to render sources outside of the active arc of the speaker layout, a virtual speaker was positioned at $180^{\circ}$ in the $0+2+0$ layout. As with the original mix, subwoofer signals for the $4+9+0$ and $0+5+0$ systems were generated using the IOSONO Core bass management system in the listening room.

The VBAP renderings were generated using the algorithms described by Pulkki [9] implemented in a realtime $\mathrm{C}++$ software rendering framework developed in the S3A project. The software takes as an input a loudspeaker configuration, the audio signals for each object, and the metadata for each object via a UDP JSON stream and outputs signals for the given loudspeaker configuration. The loudspeaker configuration is meshed into a set of triplets and a gain matrix for each triplet is inverted and multiplied by the object positions described in the metadata to calculate panning gains for each object in the scene. The calculated gains are applied to each object's audio signal to generate the output loudspeaker signals. The metadata model and the software rendering framework are described in more detail in [39].

Alongside VBAP, matrix downmixes from an objectbased $9+10+3$ render to $0+5+0$ and $0+2+0$ were also included to understand how the object-based renderings 
perform compared to standardized matrix downmix methods. The coefficients for the matrix downmixes were taken from [40]. All of the clips used in the experiment were loudness normalized using a multichannel extension to ITU-R 1770-2 [41].

\subsection{Listening Experiment}

Eight professional content producers took part in the listening experiment. Each of the participants identified that audio production was their full time profession. The participants' professional experience ranged from theater sound designer, studio manager, sound supervisor, sound editor, sound designer, sound mixer, composer, and sonic artist. The participants had experience mixing for surround systems ranging from 5.1 mixes for sport, entertainment, and theatricals to multichannel museum installations. The average age of participants was $43.5(\mathrm{SD}=9.1)$ and they were paid an honorarium for their time.

The experiment was conducted in the listening room at BBC R\&D. This listening room complies with ITU BS.1116 [42] and is equipped with 32 Genelec 8030B loudspeakers and 2 Genelec 7040 subwoofers. Further details of this listening room are given in [43].

Participants were presented with the stimuli described in Sec. 2.2 in pairs, the reference stimulus, and a version of the same content rendered for a speaker system with a lower number of channels.

For each comparison the participant was asked:

1. What they would have done differently if they had control of the version with the lower number of loudspeakers if they were mixing the clip with the aim of preserving the intent of the reference clip, and;

2. To indicate, using a list of perceptual attributes, what had changed between the reference and the downmix.

The order of the stimuli and attribute list was randomized for each participant and participants were not informed about the layout of the target reproduction systems. Participants were able to switch freely between the reference stimulus and the downmix stimulus.

The interface for the test is shown in Fig. 1. Responses to 1. were collected using open text data; the participants were asked to type each change they would make on a new line of the text box. Responses to 2 . were collected using check boxes; the attributes on the right hand side of the figure are harshness, spatial clarity, spatial naturalness, enveloping, richness of sound, ease of listening, detail, position of sound, ensemble balance, spectral resonances, clarity, realism, sense of space, spatial movement, spectral clarity, bandwidth, horizontal width, level of reverb, depth of field, amount of distortion, surrounding, subjective quality of reverb, phasiness, overall spectral balance, spatial openness, and bass. The list of attributes was taken from the findings of an elicitation experiment by Francombe et al. [31, 32]. The definitions of the attributes given in [31] were provided to the participants. Participants were also allowed to add any other attributes they perceived as having changed in an open textbox; however, none of the participants used this option suggesting that the attributes in [31] covered all of the perceptual differences in the test stimuli.

\subsection{Statistical Methods}

\subsubsection{Cluster Analysis}

Two methods of cluster analysis are used in this study$\mathrm{k}$-means and hierarchical agglomerative clustering. The aim of both of these methods is to divide a set of data into interpretable groups.

$\mathrm{K}$-means clustering is a flat clustering algorithm that aims to minimize the average squared Euclidean distance of objects from the centroid of the object's cluster [44]. In $\mathrm{k}$-means clustering, the number of clusters $k$ returned by the algorithm is specified in advance.

Hierarchical agglomerative clustering is a method of clustering whereby all of the objects begin as individual clusters. In an iterative process, the two closest clusters are merged. The final top level of the hierarchy consists of a single cluster containing all objects. Using this method, the number of clusters does not need to be specified in advance; different numbers of clusters can be obtained by slicing the hierarchical solution at different levels [45].

\subsubsection{Regression Modeling}

Categorical response variables with more than two categories can be modeled using multinomial logistic regression [46]. In the present study, the changes suggested by the sound designers for each reproduction system (see Sec. 3.1.1) can be represented in this way. Consider a multinomial categorical response variable $Y_{i}$ that can take one of $J$ values. The probability $\pi_{i, j}$ that the $i$-th response falls into the $j$-th category can be denoted as such:

$$
\pi_{i, j}=P\left(Y_{i}=j\right)
$$

In the multinomial logistic model, one of the categories of $Y_{i}$ is used as a baseline category, and the log-odds for all the other categories relative to this baseline are modeled. The odds that the $i$-th response falls into category $j$ as opposed to the baseline is $\pi_{i, j} / \pi_{i, \text { Baseline }}$.

The multinomial logistic model assumes that the logodds of each response category over the baseline follows a linear model:

$$
\ln \left(\pi_{i, j} / \pi_{i, \text { Baseline }}\right)=\alpha_{j}+\mathbf{x}_{\mathbf{i}}^{\mathbf{T}} \beta_{\mathbf{j}}
$$

where $\alpha_{j}$ is the model intercept for the $j$-th category, $\beta_{\mathbf{j}}$ is a vector of regression coefficients, $\mathbf{x}_{\mathbf{i}}$ is a vector of observations, and $\mathbf{T}$ indicates a matrix transpose. $\alpha_{j}$ and $\beta_{\mathbf{j}}$ can be estimated via maximum likelihood.

Considering Eq. (2), as the the log-odds are being modeled, taking the exponential of the $\beta_{\mathbf{j}}$ regression coefficients directly leads to an odds-ratio against the baseline category for a unit increase in the corresponding independent variable. For example, if a $\beta_{j}$ coefficient of 0.70 were found for a given binary predictor variable, this can be directly interpreted as the odds of a response occurring in the $j$-th category over the baseline category being twice as high 


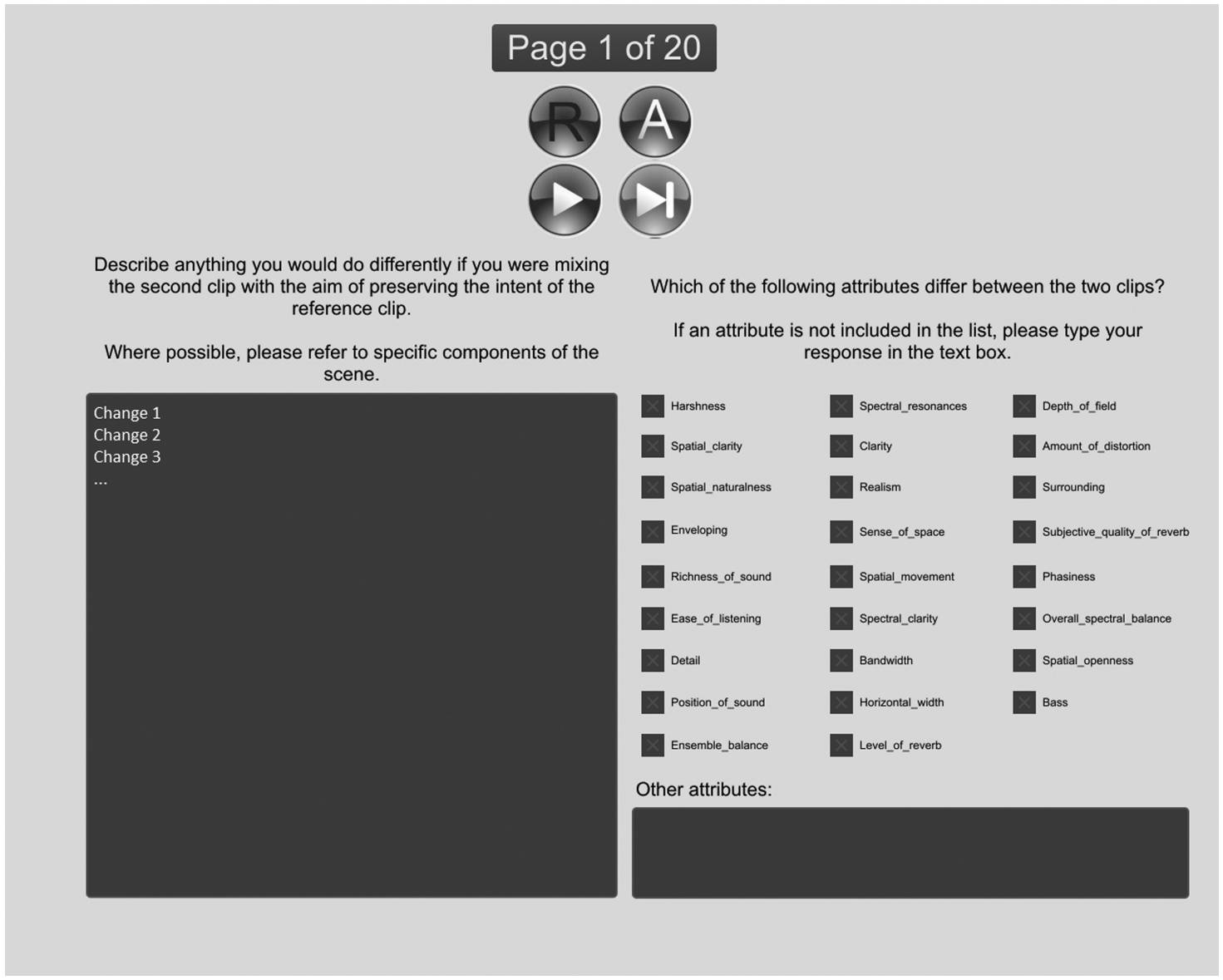

Fig. 1. Test interface used in the listening tests.

when the predictor variable takes on a value of 1 (as $e^{0.70}$ $\approx 2$ ).

Binary logistic regression, which is used in Sec. 3.4.2, can be considered as a special case of multinomial logistic regression when the number of response categories $J=2$.

\section{ANALYSIS AND RESULTS}

\subsection{Open Text Data}

\subsubsection{Text Mining}

The open text data collected using the methods described in Sec. 2.3 were initially analyzed using unsupervised text mining techniques. The aim of this exercise was to begin to look for general categories of changes that the sound designers indicated they would make to the content rendered to systems with a lower number of loudspeakers than the reference system. Text mining allows the investigation of latent structures in a collection of documents (in this case a document refers to a single phrase ${ }^{2}$ elicited in the experiment) [47]. Text mining has an advantage over simply examining word frequencies, as it can reveal clusters of phrases that relate to similar concepts; for example, in the present study the word "position" is used frequently

${ }^{2}$ In the remainder of this paper a phrase refers to a single sentence provided by the participant. in phrases relating to both static and dynamic positions of objects.

The text data were cleaned by converting every letter to lower case and by removing numerical and punctuation characters. Following this, a list of stopwords was constructed. Stopwords are words that are disregarded in the text mining process because they offer little predictive power; this may be because they are common words within the language (e.g., a, the, and) or because they are common within the domain that is being investigated (in the present study, this included words such as speaker, stereo, downmix).

A list of common English words from the SMART information retrieval project [48] was used as a base for the stopword list. This list was manually examined to ensure any words that had potential predictive power were retained. Following this, any word that only occurred once in the corpus was considered a stopword [47]. As the main aim of the exercise was to investigate mix processes, the resulting dictionary was manually examined to remove hedonic phrases (e.g., good, better, excellent) and modifiers (e.g., little, increase, decrease). The decision was made to include all audio objects identified in the phrases as stopwords to prevent the algorithm from creating clusters of objects. A list of the stopwords used is included as an appendix to this paper. Fig. 2 shows the 20 most frequently used words within the corpus after removal of stopwords. 


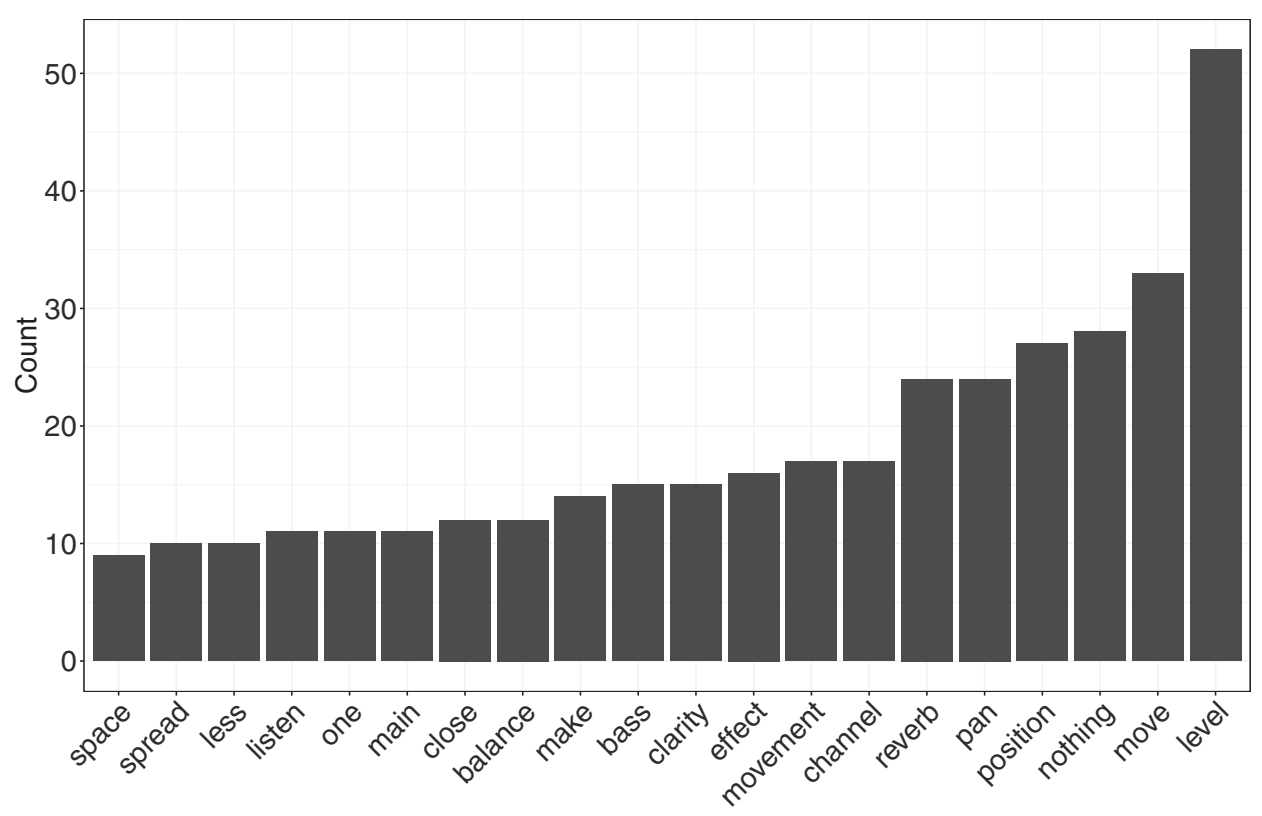

Fig. 2. Frequency of use of words within the corpus after stemming and removal of stopwords.

The text data were quantitatively represented by generating a phrase-term matrix, whereby each phrase is represented as a feature vector representing which terms in the dictionary exist in that phrase (in this context, a dictionary is a list of all words that appear in the corpus). K-means clustering (see Sec. 2.4.1) was used to analyze the phraseterm matrix. As the number of clusters $k$ is a variable that needs to be determined, a scree plot was generated to examine the within cluster sum of squares as a function of $k$. The scree plot showed no obvious elbow, but a shallowing of the gradient of the curve suggested a 20 cluster solution was an appropriate staring point. Examination of the 20 cluster solution revealed that the largest returned cluster contained a number of clear sub-clusters. Therefore, the k-means algorithm was run a second time on this top level cluster to obtain an additional 10 cluster solution making the total number of clusters 29.

Each of the clusters returned by the algorithm was automatically labelled by calculating a metric comparing mean word use for the phrases within a cluster $\left(w_{i n}\right)$ to the mean word use for the phrases not in that cluster $\left(w_{\text {out }}\right)$. The metric was calculated as follows:

$$
\begin{aligned}
& w_{\text {in }}=\frac{N_{w}}{N_{c}} \quad \text { for } w \in c \\
& w_{\text {out }}=\frac{N_{w}}{N-N_{c}} \quad \text { for } w \notin c \\
& w_{\text {diff }}=w_{\text {out }}-w_{\text {in }}
\end{aligned}
$$

where $N_{w}$ is the number of occurrences of word $w, N_{c}$ is the number of words in cluster $c$, and $N$ is the number of words in the corpus. The cluster was then automatically labelled by rank ordering $w_{\text {diff }}$ and taking the top term.

Table 2 shows the automatic labels for the first 10 phrase clusters revealed in the text mining analysis. This table also shows the number of phrases in each cluster. It can be seen
Table 2. Automatic labels for the first 10 clusters revealed in the text mining analysis.

\begin{tabular}{llc}
\hline Cluster & Automatic labels & N phrases \\
\hline 1 & Envelope & 81 \\
2 & Level & 41 \\
3 & Nothing & 27 \\
4 & Move & 26 \\
5 & Position & 24 \\
6 & Reverb & 15 \\
7 & Clarity & 14 \\
8 & Movement & 9 \\
9 & Effects & 9 \\
10 & Bass & 9 \\
\hline
\end{tabular}

that generally the automatic label describes a mix process or perceptual attribute, except the cluster labelled Nothing that consists of the responses where the participants indicated they would make no changes. From Table 2 it is apparent that a number of the clusters could be describing the same concept (for example, the clusters labelled move and position). To explore this, one-to-one follow up interviews were conducted with the test participants.

\subsubsection{Follow-Up Interviews}

The automatic labels for the clusters revealed in the text mining analysis were used in follow-up interviews with the listening test participants. In these discussions the participants were presented with the phrases in each cluster describing the suggested changes, along with the automatic key words shown in Table 2. For each cluster, they were asked to consider the following:

1. Does the cluster describe a unique category of mix process? 


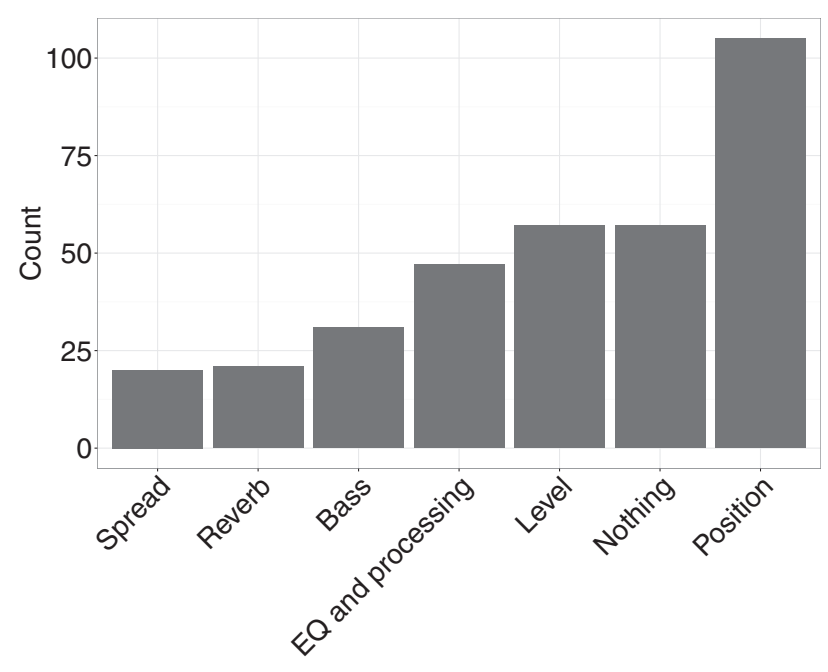

Fig. 3. Frequency of process use across all systems and program items (out of a total of 338 phrases).

2. If not, can the cluster be merged with another of the clusters?

3. Do all of the phrases belong in that cluster, or do they need to be reallocated to a different cluster?

This process was conducted iteratively, until all of the phrases in the corpus were allocated to a category.

Based on the categorizations developed by each of the participants, a phrase $x$ category matrix was built such that the matrix contained a 1 if the participant had allocated a phrase to a certain category and a 0 otherwise. Hierarchical agglomerative clustering (Ward method [49]) was conducted on these data revealing 7 clear categories:

1. Spread: A change in the spread or width of individual objects.

2. EQ and processing: Changes to frequency content and effects such as distortion.

3. Reverb: Changes to the level or time of reverb applied to individual objects.

4. Position: A change in the position or movement of individual objects.

5. Bass: A change to the level of the LFE channel.

6. Level: Changes to the level of individual objects.

7. No change: No suggested change.

The full clustering solution can be found at https://doi.org/10.17866/rd.salford.3120112.

\subsection{General Mix Processes}

Based on the clustering solution, each of the phrases in the corpus was coded according to one of the seven categories identified in Sec. 3.1.2. Fig. 3 shows the frequency of the general mix processes across all systems and program items. It can be seen from this figure that the most frequently suggested mix process was a change in position followed by no change, level, EQ and processing, bass, reverb, and spread.
Table 3. Total number of suggested changes over all participants and program items, broken down by system.

System

Total suggested changes

VBAP $4+5+0$

VBAP $0+5+0$

Matrix $0+5+0$

VBAP $0+2+0$

Matrix $0+2+0$
38

36

45

70
Table 3 shows the total number of suggested changes over all participants and program items. It can be seen from the table that the greatest number of suggested changes were for the VBAP and matrix $0+2+0$ systems ( 91 and 70 changes respectively). The distribution of these data are shown in Fig. 4, which shows the cumulative frequency of process use broken down by target reproduction system. Changes in position are the most commonly suggested mix process for the VBAP $4+5+0$, VBAP $0+5+0$ system, VBAP $0+2+0$, and matrix $0+2+0$ systems. Changes in level were the most commonly suggested mix process for the matrix $0+5+0$ system.

Fig. 5 shows the cumulative frequency of process use broken down by the type of audio object to which the process referred. The categories of objects are taken from a study into the cognitive categorization of auditory object in complex spatial audio scenes [37]. In general, for action/movement sounds, prominent transients, clear speech, and non-diegetic music, the most commonly suggested process was a change in position. For transient background sounds and sounds that indicate the presence of people, the most commonly suggested process was level. When no specific object was mentioned (i.e., processes on the overall scene), the most commonly suggested process was EQ and processing. For continuous background sounds and sounds indicating the presence of people, changes in the spread of the object were also commonly suggested.

\subsection{Attributes}

In the listening experiment described in Sec. 2.3, the participants were asked to indicate which perceptual attributes from a list had changed between the reference clip and the re-rendered or downmixed clip. Fig. 6 shows the frequency of attribute use across all systems and program items. It can be seen from this figure that the top three most frequently selected attributes relate to spatial aspects; this gives support to the finding in Sec. 3.1.1 that the most commonly suggested change was positional. The open text box to enter other attributes was not used by any of the participants.

It is likely that there is some redundancy in the attribute set, with some attributes describing the same percept. To investigate patterns in the way that the attributes were used, hierarchical agglomerative clustering (Ward method [49]) was conduced on a phrase $\mathrm{x}$ attribute matrix. A dendrogram showing the results of this analysis is shown in Fig. 7. From top to bottom, the first two clusters appear to relate to spatial attributes, the third cluster appears to relate to clarity and realism, and the fourth cluster appears to relate 


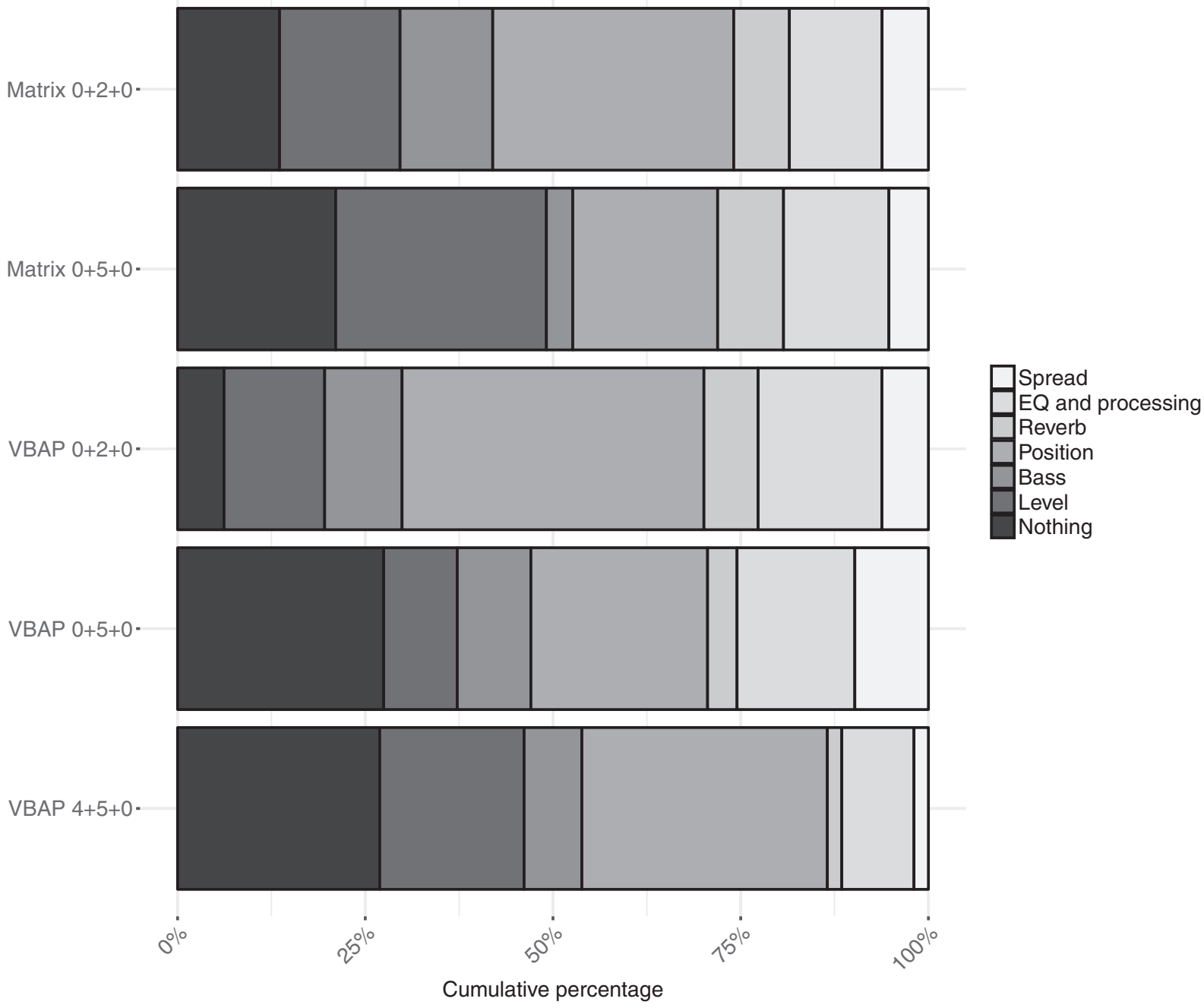

Fig. 4. Cumulative percentage of process use, broken down by system.

to artifacts. These categories of perceptual attributes will be used in the regression model described in the following section.

\subsection{Regression Models}

\subsubsection{Relationship between Perceptual Attributes and Mix Processes}

This section describes the development of a regression model to explore the relationship between the changes to perceptual attributes identified by the test participants and the changes to the mix that they suggested.

Initially, each of the perceptual attributes were included separately and individually as independent variables in a multinomial logistic regression model with the mix processes identified in Sec. 3.1.1 (Process) as a dependent variable with a baseline of No change (i.e., the model expresses the log-odds of a given mix process being suggested compared to no changes being suggested). The choice of independent and dependent variables was based on the conceptual model detailed in Sec. 1.3, which posits that changes in attributes detected by the mix engineers cause a suggestion of a mix process. The purpose of this was to identify any attributes that were not significant predictors of the changes proposed by the sound designers. The significance of each model was assessed via a likelihood ratio test against the null model. Any attribute that resulted in a likelihood ratio test with a p-value greater than 0.05 (i.e., no significant improvement in model fit over the null model) was discounted from further analysis. Of the attributes investigated, horizontal width, spectral balance, spatial openness, bandwidth, phasiness, reverb level, and spectral resonances were found not to be significant predictors of Process.

The remaining variables were added blockwise to a multinominal logistic regression model. A model containing only a variable identifying which clip was being assessed was specified as a baseline model to control for the effect of the different types of program material used in the listening experiment. The variable blocks were specified according to the results of the hierarchical cluster analysis of attribute ratings described in Sec. 3.3. The variable blocks were added to the model in the following order: 1) Clip (baseline model), 2) Spatial attributes $a, 3$ ) Spatial attributes $b$,4) Clarity attributes, and 5) Artifact attributes. 
No specific object mentioned .

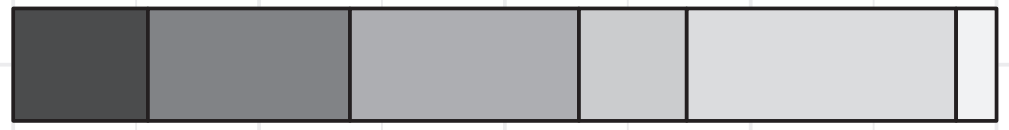

Prominent transients -

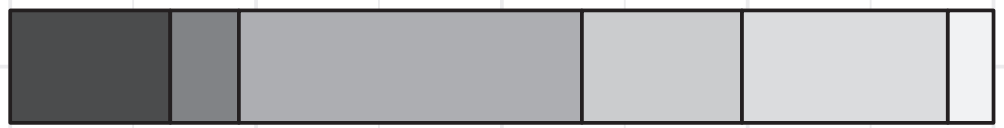

Prescence of people-

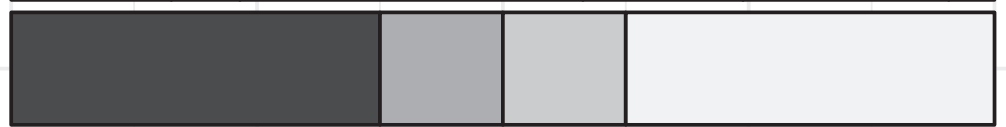

Non diegetic music -

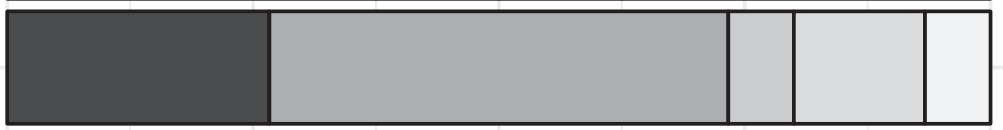

Clear speech -
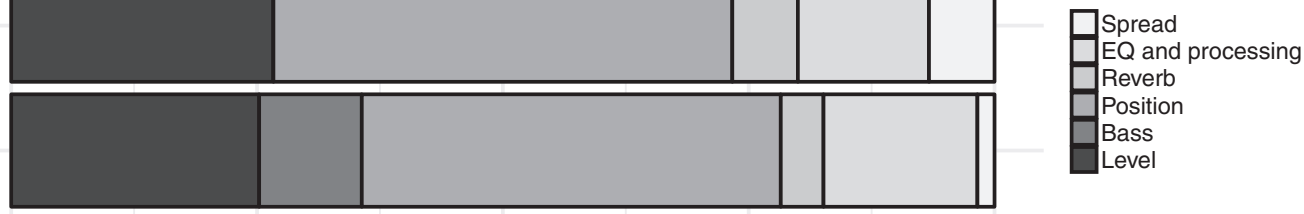

Transient background -

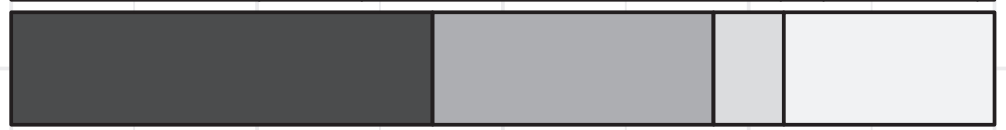

Continuous background -

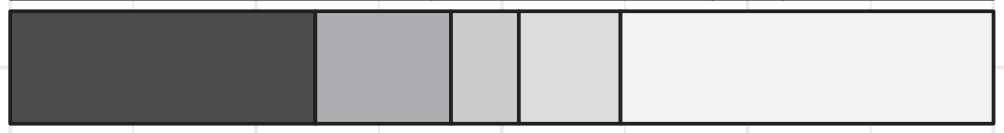

Action and movement.

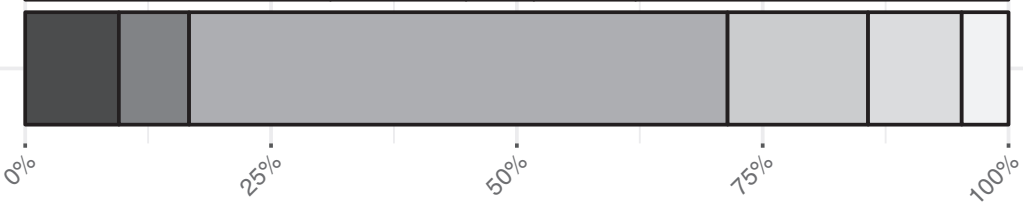

Cumulative percentage

Fig. 5. Cumulative percentage of process use broken down by object type.

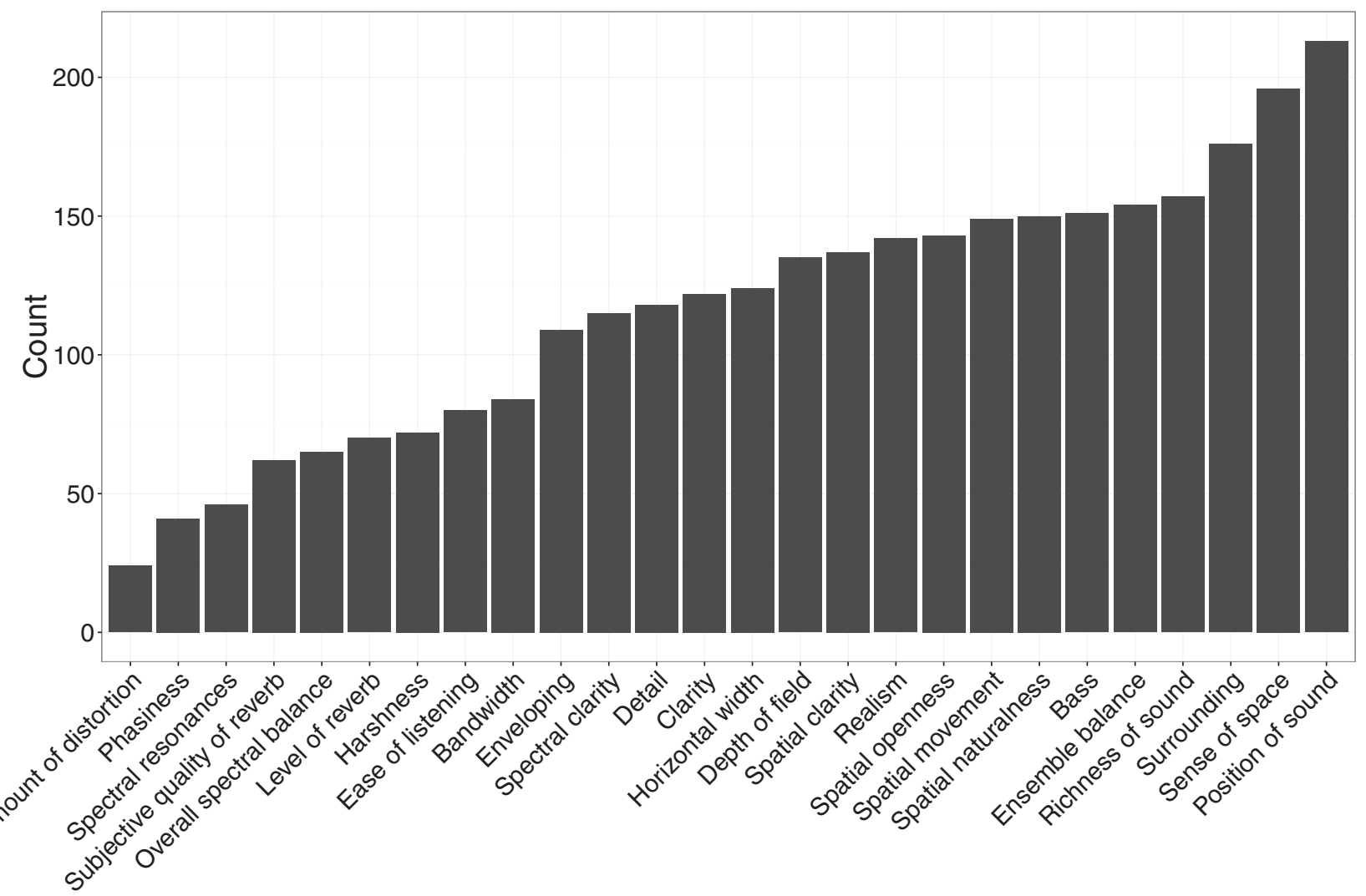

Fig. 6. Frequency of attribute use across all systems and program items. 


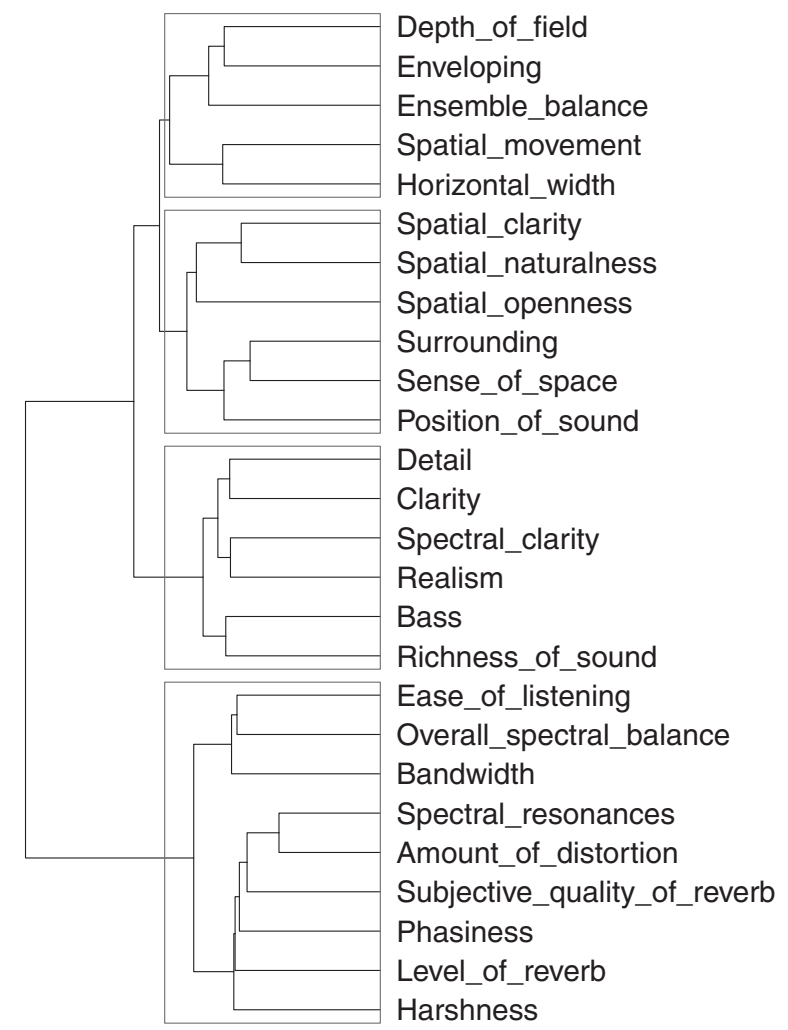

Fig. 7. Dendrogram showing hierarchical agglomerative clustering of attribute use across all systems and program items.

Table 4. Summary of the results of the multinomial logistic regression.

\begin{tabular}{lccccc}
\hline \hline Block & $\chi^{2}$ & $\Delta \chi^{2}$ & $\mathrm{p}$ & $R^{2}$ & $\Delta R^{2}$ \\
\hline Baseline & 30.4 & - & 0.03 & 0.03 & - \\
Spatial 1 & 102.25 & 71.85 & $<0.0001$ & 0.08 & 0.05 \\
Spatial 2 & 157.85 & 55.6 & $<0.0001$ & 0.13 & 0.05 \\
Timbral & 214.19 & 56.34 & $<0.0001$ & 0.18 & 0.05 \\
Artifacts & 269.49 & 55.3 & $<0.0001$ & 0.22 & 0.04 \\
\hline
\end{tabular}

The change in overall $\chi^{2}$ of the model fit was used to test the utility of each successive block of variables. The results of the multinomial logistic regression are summarized in Table 4. The table shows a clear improvement in the fit of the model as successive blocks of variables are entered into the model. The first two columns show the overall $\chi^{2}$ of the model and the change in $\chi^{2}\left(\Delta \chi^{2}\right)$ over the previous model. For each block, the change in $\chi^{2}$ over the previous model represents a significant improvement in the model fit $(\mathrm{p}<0.05)$.

The final two columns of Table 4 give the McFadden pseudo $R^{2}$ of the model and the improvement in $R^{2}$ when blocks of variables are entered into the model. The McFadden $R^{2}$ value of the full model is 0.22 . This is a pseudo $R^{2}$ based on the increase in log-likelihood of the full model over the null model. As such pseudo $R^{2}$ cannot be interpreted as a proportion of variance accounted for as in ordinary linear regression; according to [50], values between $0.2-0.4$ represent an "excellent fit."
The results of the full model are given in Table 6 of Appendix II.

\subsubsection{Relationship between Rendering System and Perceptual Attributes}

To investigate the effect of the different system types on changes to perceptual attributes, binomial logistic regression models were calculated for each of the attributes with system type as the independent variable. The models are shown in Table 5. These models are all against a baseline of the VBAP $4+5+0$ render $^{3}$, therefore the models describe the log-odds of a change of attribute being detected over the highest channel system in the test that was not the reference system. It can be seen that the only significant results at the $p<0.05$ level for the $0+5+0$ systems was for envelopment in the VBAP rendering, suggesting the two $0+5+0$ rendering methods performed similarly to the baseline VBAP $4+5+0$ system; this result is in line with recent findings from Francombe et al. [32] where little difference was found between preference ratings of 5-channel and 9-channel systems. The two $0+2+0$ systems result in significant changes in the the majority of the tested attributes.

\section{DISCUSSION}

The primary aim of the work presented in this paper was to determine a small number of common mix processes that professional content producers would employ when downmixing object-based content to different speaker layouts. Text mining of the open response data collected in the listening tests described in Sec. 2.3, combined with follow up interviews and cluster analysis, identified six general categories of mix processes. The results suggest that changes in level and position are the most commonly suggested mix processes. These results provide a framework of common processes that could be built into an object-based renderer. The next steps in this work will focus on determining quantitative rules that describe how professional mix engineers would vary these processes for different categories of audio objects.

The results presented in Fig. 5 suggest that these general processes apply differently to different categories of audio object. These findings provide evidence that any downmix operations included in an object-based audio renderer should consider the category of object to which the process is being applied. The most commonly suggested changes across all categories of objects were changes in level and position. The mix process spread was suggested more commonly for continuous and transient background objects and sounds indicating the presence of people than other object categories. Operations of the position of individual objects were commonly suggested for objects that indicate actions and movement compared to the other identified mix

\footnotetext{
${ }^{3}$ Due to the paired comparison method used, participants were not able to suggest changes to the reference system, therefore the highest channel system for which data are available is the VBAP $4+5+0$ system.
} 
Table 5. Results of multinomial regression model for Process by attributes.

\begin{tabular}{|c|c|c|c|c|}
\hline & \multicolumn{4}{|c|}{ Dependent variable vs VBAP $4+5+0$} \\
\hline & VBAP $0+5+0$ & Matrix $0+5+0$ & VBAP $0+2+0$ & Matrix $0+2+0$ \\
\hline Envelopment & $2.1462^{* *}$ & & $3.573^{* * *}$ & $2.9957^{* * *}$ \\
\hline Depth & & & $1.6704^{* * *}$ & $1.1727^{* *}$ \\
\hline Ensemble balance & & & $1.1227^{* *}$ & $1.1856^{* *}$ \\
\hline Spatial movement & & & $1.6144^{* * *}$ & $0.9345^{*}$ \\
\hline Spatial clarity & & & $1.3683^{* * *}$ & $1.5247^{* * *}$ \\
\hline Spatial naturalness & & & $2.0562^{* * *}$ & $2.1289^{* * *}$ \\
\hline Surrounding & & & $1.01881^{* *}$ & $1.0033^{* *}$ \\
\hline Sense of space & & & $1.44036^{* * *}$ & $1.26929^{* * *}$ \\
\hline Position & & & $1.4404^{* * *}$ & $1.6358^{* * *}$ \\
\hline Detail & & & $0.8633^{*}$ & $1.6084^{* * *}$ \\
\hline Spectral clarity & & $0.9708^{*}$ & $1.3319^{* *}$ & $1.1618^{* *}$ \\
\hline Clarity & & & $1.08059^{* *}$ & \\
\hline Realism & & & $1.76359^{* * *}$ & $0.88504^{*}$ \\
\hline Bass & $-0.9884^{*}$ & & $1.3077^{* * *}$ & $1.0301^{* *}$ \\
\hline Richness & & & $1.0759^{* *}$ & $1.501^{* * *}$ \\
\hline Ease of listening & & & $1.088^{*}$ & $1.381^{* *}$ \\
\hline Distortion & & & $1.3809^{*}$ & \\
\hline Quality of reverb & & & $1.8438^{* * *}$ & \\
\hline Harshness & & & $1.64^{* * *}$ & $1.39879^{* *}$ \\
\hline
\end{tabular}

processes. The process $E Q$ was suggested commonly in reference to operations on the overall scene and prominent transient sounds. One of the main advantages of objectbased audio is the ability to operate on separate objects or categories of objects. Semantic metadata could be included to indicate the category of objects, and category specific rules could be incorporated into a rendering scheme to reflect these differences. This could not be achieved with standard matrix downmixing methods.

Fig. 8 provides a summary of the regression models derived in Secs. 3.4.1 and 3.4.2; lines are drawn where significant relationships were found $(p<0.05)$ and the thickness of each line is proportional to the effect size. In this case the odds ratio has been taken as an indication of the effect size. Chen et al. [51] provide an empirical relationship between odd-ratios and Cohen's $d$. These relationships have been used to indicate the effect size in the Fig. 8; solid arrows indicate a large effect size (d 0.8), dashed arrows indicate a medium effect size $(0.4 \leqslant \mathrm{~d}<0.8)$, dotted arrows indicate a small effect size $(0.2 \leqslant \mathrm{~d}<0.4)$, and dot-dashed arrows indicate a very small effect size $(\mathrm{d} \leqslant 0.2)$.

The largest effect size for the relationships between the reproduction systems and the perceptual attributes is for the envelopment attribute, with odds ratios of 35.6 and 19.9 for the VBAP and Matrix $0+2+0$ systems respectively. This means that if the same content is rendered to $0+2+0$ using VBAP, a change in envelopment is 35.6 more likely than if the content were rendered to a $5+4+0$ system. Smaller effect sizes can be seen between the $0+2+0$ systems and the majority of the other attributes.

Overall, the largest effect sizes were with the attribute harshness followed by envelopment and ensemble balance. Recent research has shown that envelopment is one of the most important attributes in determining preference between spatial audio systems [32]. This is supported by other studies that have found that spatial attributes contribute to overall listener preference [26, 27, 52]. This suggests that OBA systems could deliver improvements to listener experience if they employed optimized rendering rules for spatial attributes such as envelopment; recent research has shown that it is possible to adapt object-based mixes to result in different levels of envelopment [53]. Relationships with large and medium effect sizes can be observed between envelopment and all of the processes apart from spread. This suggests that the test participants were able to suggest changes to ameliorate the degradation of envelopment. Other attributes exhibiting large and medium effect sizes include ensemble balance, spatial movement, spatial naturalness, clarity, bass, richness, and harshness.

\section{CONCLUSION}

This paper has presented the results of an experiment designed to identify a small number of the most common mix processes used by sound designers when mixing objectbased content to loudspeaker systems with different numbers of channels. Six general mix processes were identified from open text data gathered in the listening experiment. These processes were related to the spatial spread of individual objects, EQ and processing, reverb, position, bass, and level. Over all program items and systems tested, the most commonly suggested process was a change in the position of individual objects.

Multinomial and binary logistic regression models were developed to investigate the relationships between the type of reproduction system, changes in perceptual attributes, and suggested mix processes. Significant relationships were found between the 2-channel stereo systems tested and the majority of the perceptual attributes investigated in the experiment; the largest effect size was between the VBAP 


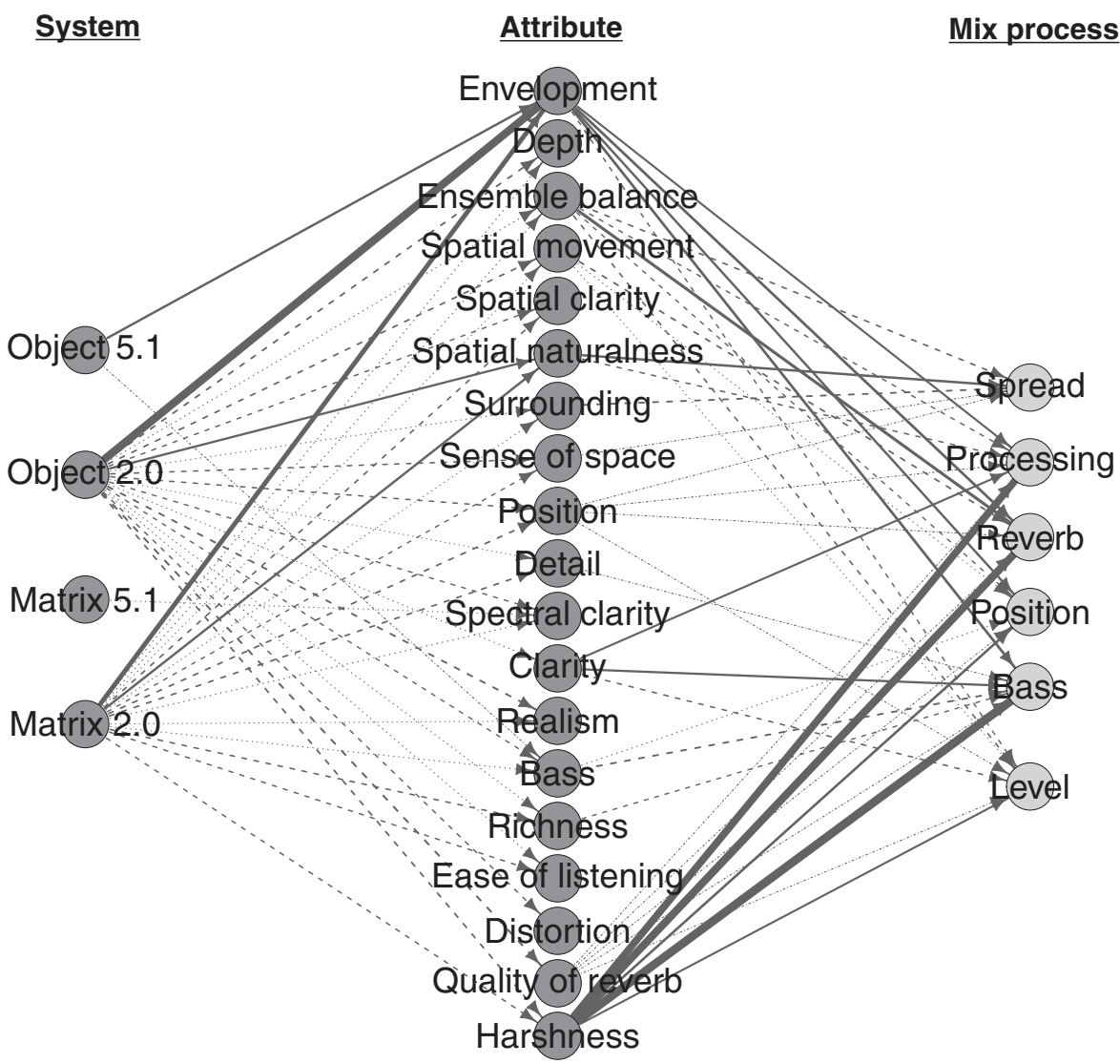

Fig. 8. Summary of the relationships revealed in models described in Secs. 3.4.1 and 3.4.2. The solid arrows indicate a large effect size, dashed is a medium effect size, dotted is a small effect size, and dot-dahed is a very small effect size.

$0+2+0$ system and envelopment. Significant relationships were also found between changes in perceptual attributes and the suggested mix processes. The largest effect sizes were with the attribute harshness followed by envelopment and ensemble balance. Although it was ensured that the program material used in the test covered a wide range of audio object types, it should be noted that the study was limited to audio drama program material; other processes and relationships may be found for different types of program material.

Taken together, these results show that when professional content producers detect changes in perceptual attributes when rendering the same content to systems with different numbers of channels, they are able to suggest changes to the mix that can be categorized into a small number of common processes. The relative frequency that the content creators suggested different mix processes was found to differ between categories of audio objects; this finding suggests any downmix operations included in an object-based audio renderer should consider the category of object to which the process is being applied. In the study, test participants were asked about their intentions rather than asking them to directly operate on the mix. The next step of this work will be to allow content producers to directly manipulate the identified mix processes in a method of adjustment task in order to determine quantitative rules that could be built into an intelligent object-based renderer.

\section{ACKNOWLEDGMENTS}

This work was supported by the EPSRC Programme Grant S3A: Future Spatial Audio for an Immersive Listener Experience at Home (EP/L000539/1) and the BBC as part of the BBC Audio Research Partnership. The authors would like to thank Chris Pike from BBC R\&D for his help generating the the material for the tests and Jon Francombe from the University of Surrey for his input and comments on the design of the experiment. Finally, the authors would like to thank the participants of the experiment for their time.

The experimental data underlying the findings are fully available without restriction, details are available from https://doi.org/10.17866/rd.salford.3120112.

\section{REFERENCES}

[1] M. Armstrong, M. Brooks, A. Churnside, M. Evans, F. Melchior, and M. Shotton "Object-Based Broadcasting-Curation, Responsiveness and User Experience," International Broadcasting Convention, IBC2014 (2014).

[2] S3A - Future Spatial Audio for an Immersive Listener Experience, http://www.s3a-spatialaudio.org/wordpress/. Accessed: 2017-08-06.

[3] Orpheus - Object-Based Audio Experience, https://orpheus-audio.eu/. Accessed: 2017-08-06. 
[4] 2-Immerse, https://2immerse.eu/. Accessed: 201708-06.

[5] J. Herre, J. Hilpert, A. Kuntz, and J. Plogsties "MPEG-H 3D Audio-The New Standard for Coding of Immersive Spatial Audio," IEEE J. Sel. Topics Signal Process., vol. 9, no. 5, pp. 770-779 (2015 May), https://doi.org/10.1109/jstsp.2015.2411578.

[6] S. Füg, D. Marston, and S. Norcross "The Audio Definition Mode-A Flexible Standardized Representation for Next Generation Audio Content in Broadcasting and Beyond," presented at the 141st Convention of the Audio Engineering Society (2016 Sep.), convention paper 9626.

[7] S. Mehta, T. Onders, and J. Riedmiller "Recipes for Creating and Delivering Next-Generation Broadcast Audio," SMPTE Motion Imaging J., vol. 125, no. 9, pp. 25-32 (2015 Nov.-Dec.), https://dx.doi.org/10.5594/ JMI.2016.2619304.

[8] J. M. Jot, B. Smith, and J. Thompson "Dialog Control and Enhancement in Object-Based Audio Systems," presented at the 139th Convention of the Audio Engineering Society (2015 Oct.), convention paper 9356.

[9] V. Pulkki "Virtual Sound Source Positioning Using Vector Base Amplitude Panning," J. Audio Eng. Soc., vol. 45, pp. 456-466 (1997 Jun.).

[10] D. Dugan “Automatic Microphone Mixing," J. Audio Eng. Soc., vol. 23 no. 6 pp. $442-449$ (1975 Aug.).

[11] M. J. Terrell and J. D. Reiss "Automatic Monitor Mixing for Live Musical Performance," J. Audio Eng. Soc., vol. 57, pp. 927-936 (2009 Nov.).

[12] E. Perez-Gonzalez and J. D. Reiss "Automatic Mixing: Live Downmixing Stereo Panner," Proc. 10th Int. Conf. Digital Audio Effects (DAFx-07), Bordeaux, France (2007 Sep.).

[13] J. D. Reiss and E. Perez-Gonzalez "An Automatic Maximum Gain Normalization Technique with Applications to Audio Mixing," presented at the 124th Convention of the Audio Engineering Society (2008 May), convention paper 7411 .

[14] E. Perez-Gonzalez and J. D. Reiss "Improved Control for Selective Minimization of Masking Using InterChannel Dependancy Effects," Proc. 11th Int. Conf. Digital Audio Effects (DAFx-08), Espoo, Finland (2008 Sep.).

[15] S. Hafezi and J. D. Reiss "Autonomous Multitrack Equalization Based on Masking Reduction," J. Audio Eng. Soc., vol. 63, pp. 312-323 (2015 May).

[16] B. Kolasinski "A Framework for Automatic Mixing Using Timbral Similarity Measures and Genetic Optimization," presented at the 124th Convention of the Audio Engineering Society (May 2008), convention paper 7496.

[17] S. Mansbridge, S. Finn, and J. D. Reiss "Implementation and Evaluation of Autonomous Multi-Track Fader Control," presented at the 132nd Convention of the Audio Engineering Society (2012 Apr.), convention paper 8588 .

[18] G. Ren, G. Bocko, J. Lundberg, D. Headlam, and M. F. Bocko "Automatic Music Production System Employing Probabilistic Expert Systems," presented at the 129th Convention of the Audio Engineering Society (2010 Nov.), convention paper 8255 .
[19] P. Pestana and J. D. Reiss "Intelligent Audio Production Strategies Informed by Best Practices," presented at the AES 53rd International Conference: Semantic Audio (2014 Jan.), conference paper S2-2.

[20] T. Letowski "Sound Quality Assessment: Concepts and Criteria," presented at the 87th Convention of the Audio Engineering Society (1989 Oct.), convention paper 2825 .

[21] F. Rumsey, S. Zieliński, R. Kassier, and S. Bech "On the Relative Importance of Spatial and Timbral Fidelities in Judgments of Degraded Multichannel Audio Quality," J. Acoust. Soc. of Amer., vol. 118, no. 2, pp. 968-976 ( 2005 Aug.) https://doi.org/10.1121/1.1945368.

[22] N. Zacharov and K. Koivuniemi "Unravelling the Perception of Spatial Sound Reproduction: Analysis \& External Preference Mapping," presented at the 111th Convention of the Audio Engineering Society (2001 Nov.), convention paper 5423.

[23] N. Zacharov and K. Koivuniemi "Unravelling the Perception of Spatial Sound Reproduction: Language Development, Verbal Protocol Analysis and Listener Training," presented at the 111th Convention of the Audio Engineering Society (2001 Nov.), convention paper 5424

[24] N. Zacharov and K. Koivuniemi "Unraveling the Perception of Spatial Sound Reproduction: Techniques and Experimental Design," presented at the AES 19th International Conference: Surround Sound-Techniques, Technology, and Perception (2001 Jun.), conference paper 1929.

[25] C. Guastavino and B. F. G. Katz "Perceptual Evaluation of Multi-Dimensional Spatial Audio Reproduction," J. Acoust. Soc. of Amer., vol. 116, no. 2, pp. 1105-1115 (2004 Aug.) https://doi.org/10.1121/1.1763973.

[26] S. Choisel and F. Wickelmaier "Extraction of Auditory Features and Elicitation of Attributes for the Assessment of Multichannel Reproduced Sound," J. Audio Eng. Soc., vol. 54, pp. 815-826 (2006 Sep.).

[27] S. Choisel and F. Wickelmaier "Evaluation of Multichannel Reproduced Sound: Scaling Auditory Attributes Underlying Listener Preference," J. Acoust. Soc. of Amer., vol. 121, no. 1, pp. 388-400 (2007 Jan.) https://doi.org/10.1121/1.2385043.

[28] A. Lindau, V. Erbes, S. Lepa, H. J. Maempel, F. Brinkman, and S. Weinzierl "A Spatial Audio Quality Inventory (SAQI)," Acta Acust. united Acust., vol. 100, no. 5, pp. 984-994 (2014 Sep.-Oct.) https://doi.org/10.3813/AAA.918778.

[29] N. Zacharov, T. Pedersen and C. Pike "A Common Lexicon for Spatial Sound Quality Assessment-Latest Developments," Proc. 8th Int. Conf. on Quality of Multimedia Experience (QoMEX), Lisbon, Portugal (2016 Jun.) https://doi.org/10.1109/QoMEX.2016.7498967.

[30] J. Francombe, T. Brookes, and R. Mason "Perceptual Evaluation of Spatial Audio: Where Next?" Proc. 22nd Int. Congress on Sound and Vibration (ICSV) Florence, Italy (2015 Jul.).

[31] J. Francombe, T. Brookes, and R. Mason "Evaluation of Spatial Audio Reproduction Methods (Part 1): 
Elicitation of Perceptual Differences," J. Audio Eng. Soc., vol. 65, pp. 198-211 (2017 Mar.).

[32] J. Francombe, T. Brookes, R. Mason, and J. Woodcock "Evaluation of Spatial Audio Reproduction Methods (Part 2): Analysis of Listener Preference," J. Audio Eng. Soc., vol. 65, pp. 212-225 (2017 Mar.).

[33] ITU-R, "Recommendation BS.2051, Advanced sound system for programme production," International Telecommunications Union (ITU) (2017).

[34] C. H. Coombs A Theory of Data (Wiley, 1964).

[35] J. Blauert and U. Jekosch "A Layer Model of Sound Quality,” J. Audio Eng. Soc., vol. 60, pp. 4-12 (2012 Jan./Feb.).

[36] J. Woodcock, C. Pike, F. Melchior, P. Coleman, A. Franck, and A. Hilton "Presenting the S3A Object-Based Audio Drama Dataset," presented at the 140th Convention of the Audio Engineering Society (2016 May), eBrief 255.

[37] J. Woodcock, W. J. Davies, T. J. Cox, and F. Melchior "Categorization of Broadcast Audio Objects in Complex Auditory Scenes," J. Audio Eng. Soc., vol. 64, pp. 380-394 (2016 Jun.).

[38] J. Woodcock, W. J. Davies, and T. J. Cox "A Cognitive Framework for the Categorization of Auditory Objects in Urban Soundscapes," Appl. Acoust., vol. 121, pp. 56-64 (2017 Jun.) https://doi.org/10.1016/j.apacoust.2017.01.027.

[39] P. Coleman, A. Franck, J. Francombe, Q. Liu, T. Campos, R. J. Hughes, D. Menzies, M. F. S. Gálvez, Y. Tang, J. Woodcock, P. J. B. Jackson, F. Melchior, C. Pike, F. M. Fazi, T. J. Cox, and A. Hilton "An Audio-Visual System for Object-Based Audio: From Recording to Listening," IEEE Tran. Mult. (In Press, 2018).

[40] T. Komori, S. Oode, K. Ono, K. Irie, Y. Sasaki, T. Hasegawa, and I. Samaya "Subjective Loudness of 22.2 Multichannel Programs," presented at the 138th Convention of the Audio Engineering Society (May 2015), convention paper 9219.

[41] ITU-R, "Recommendation BS.1770-2, Algorithms to measure audio programme loudness and true-peak audio level," International Telecommunications Union (ITU) (2011).

[42] ITU-R, "Recommendation BS.1116, Methods for the subjective assessment of small impairments in audio systems including multichannel sound systems," International Telecommunications Union (1997).

[43] T. Nixon, A. Bonny, and F. Melchior "A Reference Listening Room for 3D Audio Research," Proc. 3rd Int. Conf. on Spatial Audio (ICSA), Graz, Austria (2015 Sep.).

[44] C. D. Manning, P. Raghavan, and H. Schütz Introduction to Information Retrieval, Volume 1 (Cambridge University Press, 2008).

[45] R. Xu and D. Wunch Clustering (John Wiley \& Sons, 2008), pp. 31-61.

[46] J. S. Long and J. Freese Regression Models for Categorical Dependent Variables Using Stata (Stata Press, 2006).
[47] S. M. Weiss, N. Indurkhya, T. Zhang, and F. Damerau Text Mining: Predictive Methods for Analyzing Unstructured Information (Springer Science \& Business Media, 2010).

[48] C. Buckley "Implementation of the Smart Information Retrieval System, " Technical Report, Cornell University (1985).

[49] J. H. Ward "Hierarchical Grouping to Optimize an Objective Function,” J. Am. Stat. Assoc., vol. 58, no. 301, pp. 236-244 (1963 Mar.).

[50] D. McFadden "Quantitative Methods for Analyzing Travel Behavior of Individuals: Some Recent Developments," in Behavioural Travel Modelling (Croom Helm, 1978), pp. 279-318.

[51] H. Chen, P. Cohen, and S. Chen "How Big Is a Big Odds Ratio? Interpreting the Magnitudes of Odds Ratios in Epidemiological Studies," Communications in Statistics-Simulation and Computation, vol. 39, no. 4, pp. 860-864 (2010 Apr.) https://doi.org/10.1080/ 03610911003650383.

[52] N. Zacharov and K. Koivuniemi "Audio Descriptive Analysis \& Mapping of Spatial Sound Displays," Proc. 2001 Int. Conf. on Auditory Display, Espoo, Finland (2001 Jul.).

[53] J. Francombe, T. Brookes, and R. Mason "Determination and Validation of Mix Parameters for Modifying Envelopment in Object-Based Audio Mixes," J. Audio Eng. Soc. (In press).

\section{APPENDIX I}

Below is a list of the stopwords used in the text mining analysis:

doesnt, sound, sounds, bit, think, also, downmix, good, better, slightly, seems, there, needs, little, get, like, feels, mix, clips, clip, lack, increase, decrease, lower, raise, slight, better, thinner, nice, perfect, cleaner, very, low, sudden, slight, too, excllent, reduce, noticible, previous, add, between, sides, down, sources, front, rear, left, right, above, central, surround, actors, hubub, man, child, Children, vo, dialogue, loudhailer, lady, kid, Voice, crowd, dog, bark, violins, music, chord, narrator, narrators, woodpecker, megaphone, dad, humming, chartacters, speach, monster, son, girls, tom, shouting, boy, ducks, synth, dinosaur, dialogues, barking, footsteps, male, vocal, girl, speach, megafono, thump, goose, character, background speakers, speaker, far, match, original, wider, centre, trebly, first, half, opening, back, harsh, mid, high, field, shifted, atmos, stereo, lost, voice, well

\section{APPENDIX II}

Table 6 shows the results of the model described in Sec. 3.4.1. The un-bracketed values show the model coefficients and the bracketed values show the standard errors. In this model, a large coefficient means a greater chance that a mix process would be used if a change in the attribute was detected. 
Table 6. Results of multinomial regression model for Process by Clip and attributes.

\begin{tabular}{|c|c|c|c|c|c|c|}
\hline & \multicolumn{6}{|c|}{ Dependent variable vs No change } \\
\hline & Spread & Processing & Reverb & Position & Bass & Level \\
\hline Clip2:Clip1 & $\begin{array}{l}3.522^{* *} \\
(1.532)\end{array}$ & $\begin{array}{c}2.043^{* *} \\
(0.831)\end{array}$ & $\begin{array}{c}1.877 \\
(1.212)\end{array}$ & $\begin{array}{l}1.698^{* *} \\
(0.752)\end{array}$ & $\begin{array}{c}2.376^{* *} \\
(1.052)\end{array}$ & $\begin{array}{c}0.343 \\
(0.798)\end{array}$ \\
\hline Clip3:Clip1 & $\begin{array}{r}2.588^{*} \\
(1.396)\end{array}$ & $\begin{array}{c}0.537 \\
(0.724)\end{array}$ & $\begin{array}{c}-0.450 \\
(1.283)\end{array}$ & $\begin{array}{l}1.327^{* *} \\
(0.628)\end{array}$ & $\begin{array}{l}2.214^{* *} \\
(0.947)\end{array}$ & $\begin{array}{c}0.222 \\
(0.641)\end{array}$ \\
\hline Clip4:Clip1 & $\begin{array}{c}2.540^{*} \\
(1.500)\end{array}$ & $\begin{array}{c}-0.308 \\
(0.761)\end{array}$ & $\begin{array}{c}0.332 \\
(1.179)\end{array}$ & $\begin{array}{c}0.500 \\
(0.651)\end{array}$ & $\begin{array}{c}1.256 \\
(0.997)\end{array}$ & $\begin{array}{c}0.110 \\
(0.618)\end{array}$ \\
\hline Envelopment & $\begin{array}{c}1.286 \\
(0.936)\end{array}$ & $\begin{array}{l}1.962^{* *} \\
(0.787)\end{array}$ & $\begin{array}{c}2.302^{* *} \\
(1.010)\end{array}$ & $\begin{array}{l}2.124^{* * *} \\
(0.709)\end{array}$ & $\begin{array}{l}2.285^{* * *} \\
(0.869)\end{array}$ & $\begin{array}{c}1.340^{*} \\
(0.756)\end{array}$ \\
\hline Depth & $\begin{array}{c}-0.269 \\
(0.826)\end{array}$ & $\begin{array}{c}-1.106 \\
(0.687)\end{array}$ & $\begin{array}{c}0.193 \\
(0.899)\end{array}$ & $\begin{array}{r}-0.243 \\
(0.556)\end{array}$ & $\begin{array}{c}-0.419 \\
(0.754)\end{array}$ & $\begin{array}{c}0.256 \\
(0.600)\end{array}$ \\
\hline Ensemble balance & $\begin{array}{l}1.562^{* *} \\
(0.772)\end{array}$ & $\begin{array}{l}1.344^{* *} \\
(0.594)\end{array}$ & $\begin{array}{l}2.446^{* * *} \\
(0.864)\end{array}$ & $\begin{array}{l}1.308^{* * *} \\
(0.503)\end{array}$ & $\begin{array}{c}0.489 \\
(0.673)\end{array}$ & $\begin{array}{l}1.504^{* * *} \\
(0.529)\end{array}$ \\
\hline Spatial movement & $\begin{array}{c}1.223 \\
(0.823)\end{array}$ & $\begin{array}{c}0.544 \\
(0.607)\end{array}$ & $\begin{array}{l}1.649^{* *} \\
(0.806)\end{array}$ & $\begin{array}{l}1.089^{* *} \\
(0.534)\end{array}$ & $\begin{array}{c}-0.133 \\
(0.712)\end{array}$ & $\begin{array}{c}0.980^{*} \\
(0.581)\end{array}$ \\
\hline Spatial clarity & $\begin{array}{c}0.348 \\
(0.936)\end{array}$ & $\begin{array}{c}0.216 \\
(0.692)\end{array}$ & $\begin{array}{c}-0.142 \\
(0.914)\end{array}$ & $\begin{array}{c}-0.223 \\
(0.591)\end{array}$ & $\begin{array}{r}-0.329 \\
(0.801)\end{array}$ & $\begin{array}{c}-0.601 \\
(0.645)\end{array}$ \\
\hline Spatial naturalness & $\begin{array}{l}2.156^{* *} \\
(0.940)\end{array}$ & $\begin{array}{l}1.435^{* *} \\
(0.688)\end{array}$ & $\begin{array}{c}0.787 \\
(0.878)\end{array}$ & $\begin{array}{c}0.577 \\
(0.573)\end{array}$ & $\begin{array}{c}0.233 \\
(0.781)\end{array}$ & $\begin{array}{c}0.792 \\
(0.618)\end{array}$ \\
\hline Surrounding & $\begin{array}{c}1.711^{*} \\
(1.023)\end{array}$ & $\begin{array}{c}-0.452 \\
(0.663)\end{array}$ & $\begin{array}{c}0.459 \\
(0.948)\end{array}$ & $\begin{array}{c}0.509 \\
(0.589)\end{array}$ & $\begin{array}{r}-0.203 \\
(0.750)\end{array}$ & $\begin{array}{c}0.235 \\
(0.622)\end{array}$ \\
\hline Sense of space & $\begin{array}{r}-1.845^{*} \\
(1.079)\end{array}$ & $\begin{array}{c}-0.628 \\
(0.689)\end{array}$ & $\begin{array}{c}-1.223 \\
(1.023)\end{array}$ & $\begin{array}{c}-0.359 \\
(0.619)\end{array}$ & $\begin{array}{c}-0.894 \\
(0.761)\end{array}$ & $\begin{array}{c}-0.683 \\
(0.656)\end{array}$ \\
\hline Position & $\begin{array}{c}-1.915^{* *} \\
(0.873)\end{array}$ & $\begin{array}{c}-1.573^{* *} \\
(0.675)\end{array}$ & $\begin{array}{c}-2.058^{* *} \\
(0.915)\end{array}$ & $\begin{array}{r}-0.756 \\
(0.567)\end{array}$ & $\begin{array}{r}-1.050 \\
(0.714)\end{array}$ & $\begin{array}{c}-1.471^{* *} \\
(0.589)\end{array}$ \\
\hline Detail & $\begin{array}{c}-0.231 \\
(0.959)\end{array}$ & $\begin{array}{r}-0.929 \\
(0.790)\end{array}$ & $\begin{array}{r}-1.650 \\
(1.097)\end{array}$ & $\begin{array}{r}-0.396 \\
(0.669)\end{array}$ & $\begin{array}{c}-1.858^{* *} \\
(0.943)\end{array}$ & $\begin{array}{c}-0.487 \\
(0.738)\end{array}$ \\
\hline Spectral clarity & $\begin{array}{c}0.076 \\
(0.825)\end{array}$ & $\begin{array}{c}0.611 \\
(0.693)\end{array}$ & $\begin{array}{c}-0.669 \\
(0.991)\end{array}$ & $\begin{array}{c}0.701 \\
(0.590)\end{array}$ & $\begin{array}{c}0.107 \\
(0.843)\end{array}$ & $\begin{array}{c}0.353 \\
(0.633)\end{array}$ \\
\hline Clarity & $\begin{array}{c}-0.884 \\
(1.056)\end{array}$ & $\begin{array}{l}1.942^{* * *} \\
(0.737)\end{array}$ & $\begin{array}{c}1.409 \\
(0.946)\end{array}$ & $\begin{array}{c}0.321 \\
(0.666)\end{array}$ & $\begin{array}{l}2.043^{* *} \\
(0.833)\end{array}$ & $\begin{array}{l}1.464^{* *} \\
(0.667)\end{array}$ \\
\hline Realism & $\begin{array}{c}1.311 \\
(0.882)\end{array}$ & $\begin{array}{c}1.168 \\
(0.739)\end{array}$ & $\begin{array}{c}0.059 \\
(0.992)\end{array}$ & $\begin{array}{c}0.825 \\
(0.642)\end{array}$ & $\begin{array}{c}0.195 \\
(0.847)\end{array}$ & $\begin{array}{c}0.262 \\
(0.696)\end{array}$ \\
\hline Bass & $\begin{array}{c}0.803 \\
(0.814)\end{array}$ & $\begin{array}{c}0.712 \\
(0.648)\end{array}$ & $\begin{array}{c}0.884 \\
(0.885)\end{array}$ & $\begin{array}{l}1.115^{* *} \\
(0.549)\end{array}$ & $\begin{array}{l}1.743^{* *} \\
(0.779)\end{array}$ & $\begin{array}{c}0.900 \\
(0.617)\end{array}$ \\
\hline Richness & $\begin{array}{c}-0.458 \\
(0.806)\end{array}$ & $\begin{array}{c}0.237 \\
(0.666)\end{array}$ & $\begin{array}{c}0.962 \\
(0.842)\end{array}$ & $\begin{array}{c}0.066 \\
(0.580)\end{array}$ & $\begin{array}{r}1.278^{*} \\
(0.729)\end{array}$ & $\begin{array}{c}0.242 \\
(0.629)\end{array}$ \\
\hline Ease of listening & $\begin{array}{c}-0.324 \\
(1.123)\end{array}$ & $\begin{array}{c}-1.012 \\
(0.868)\end{array}$ & $\begin{array}{c}-0.642 \\
(1.285)\end{array}$ & $\begin{array}{c}0.368 \\
(0.749)\end{array}$ & $\begin{array}{c}-1.115 \\
(1.032)\end{array}$ & $\begin{array}{c}-0.001 \\
(0.783)\end{array}$ \\
\hline Amount of distortion & $\begin{array}{c}-0.366 \\
(1.678)\end{array}$ & $\begin{array}{c}0.114 \\
(1.243)\end{array}$ & $\begin{array}{c}-0.073 \\
(1.433)\end{array}$ & $\begin{array}{c}-0.867 \\
(1.163)\end{array}$ & $\begin{array}{c}0.078 \\
(1.452)\end{array}$ & $\begin{array}{c}-0.241 \\
(1.183)\end{array}$ \\
\hline Quality of reverb & $\begin{array}{c}-0.054 \\
(0.908)\end{array}$ & $\begin{array}{c}-2.800^{* * * *} \\
(0.842)\end{array}$ & $\begin{array}{r}-1.816^{*} \\
(0.957)\end{array}$ & $\begin{array}{c}-2.355^{* * *} \\
(0.724)\end{array}$ & $\begin{array}{c}-2.416^{* * *} \\
(0.917)\end{array}$ & $\begin{array}{c}-1.848^{* *} \\
(0.752)\end{array}$ \\
\hline Harshness & $\begin{array}{c}1.723 \\
(1.222)\end{array}$ & $\begin{array}{l}3.484^{* * *} \\
(1.018)\end{array}$ & $\begin{array}{l}3.548^{* * *} \\
(1.136)\end{array}$ & $\begin{array}{l}2.214^{* *} \\
(0.984)\end{array}$ & $\begin{array}{l}3.647^{* * *} \\
(1.068)\end{array}$ & $\begin{array}{c}1.934^{*} \\
(1.018)\end{array}$ \\
\hline Constant & $\begin{array}{c}-5.186^{* * *} \\
(1.469)\end{array}$ & $\begin{array}{c}-1.994^{* * *} \\
(0.601)\end{array}$ & $\begin{array}{c}-3.632^{* * *} \\
(0.992)\end{array}$ & $\begin{array}{c}-2.207^{* * *} \\
(0.553)\end{array}$ & $\begin{array}{c}-2.970^{* * *} \\
(0.847)\end{array}$ & $\begin{array}{c}-1.121^{* *} \\
(0.466)\end{array}$ \\
\hline
\end{tabular}

${ }^{*} \mathrm{p}<0.1 ;{ }^{* *} \mathrm{p}<0.05 ;{ }^{* * *} \mathrm{p}<0.01$ 


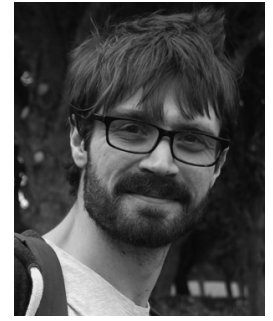

James Woodcock

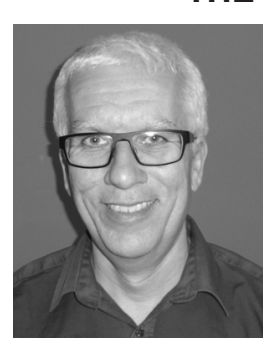

William J. Davies

\section{THE AUTHORS}

James Woodcock is a research fellow at the University of Salford. His primary area of research is the perception and cognition of complex sound and vibration. James holds a B.Sc. in audio technology, a M.Sc. by research in product sound quality, and a Ph.D. in the human response to whole body vibration, all from the University of Salford. James is currently working on the EPSRC funded S3A project. His work on this project mainly focuses on the perception of auditory objects in complex scenes, the listener experience of spatial audio, and intelligent rendering for object-based audio.

Bill Davies is professor of acoustics and perception at the University of Salford. He researches human response to complex sound fields in areas such as room acoustics, spatial audio, and urban soundscapes. He led the Positive Soundscape Project, an interdisciplinary effort to develop new ways of evaluating the urban sound environment. Bill also leads work on perception of complex auditory scenes on the S3A project. He edited a special edition of Applied Acoustics on soundscapes, and sits on ISO TC43/SC1/WG54 producing standards on soundscape assessment. He is an Associate Dean in the School of Computing, Science and Engineering at Salford and a recent Vice-President of the Institute of Acoustics (the UK professional body). Bill holds a B.Sc. in electroacoustics and a $\mathrm{Ph} . \mathrm{D}$. in auditorium acoustics, both from Salford. He is the author of 80 academic publications in journals, conference proceedings, and books.

Frank Melchior received the Dipl.-Ing. degree in media technology from the Ilmenau University of Technology,

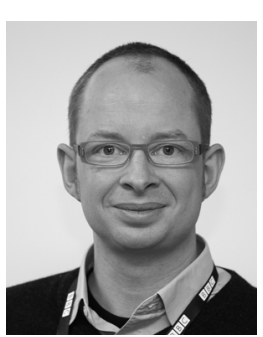

Frank Melchior

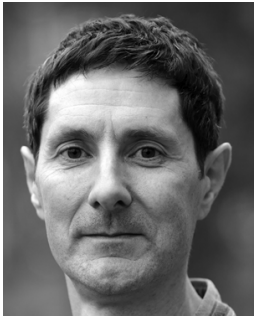

Trevor J. Cox
Germany, in 2003 and the Dr ing. degree from Delft University of Technology, The Netherlands, in 2011. From 2012 to 2017 he led the audio research group and the BBC Audio Research Partnership at BBC Research and Development. From 2009 to 2012 he was the Chief Technical Officer and Director Research and Development at IOSONO GmbH, Germany. From 2003 to 2009 he worked as a researcher at the Fraunhofer Institute Digital Media Technology, Germany. He holds several patents and has authored and coauthored a number of papers in international journals and conference proceedings.

Trevor Cox is Professor of acoustic engineering at the University of Salford and a past president of the UK's Institute of Acoustics (IOA). Trevor's diffuser designs can be found in rooms around the world. He is co-author of Acoustic Absorbers and Diffusers. He was award the IOA's Tyndall Medal in 2004. He is currently working on two major audio projects. Making Sense of Sound is a Big Data project that combines perceptual testing and machine learning. S3A is investigating future technologies for spatial audio in the home. Trevor was given the IOA award for promoting acoustics to the public in 2009. He has presented science shows at the Royal Albert Hall, Purcell Rooms, and Royal Institution. Trevor has presented 24 documentaries for BBC radio including: "The Physicist's Guide to the Orchestra." For his popular science book Sonic Wonderland (in USA: The Sound Book), he won an ASA Science Writing Award in 2015. His second popular science book Now You're Talking will be published in May 2018. @ trevor_cox 